\title{
General medical training in gastroenterology: views from specialist trainees on the challenges of dual accreditation 垱”
}

\author{
Authors: James R Neale ${ }^{A}$ and Peter J Basford, ${ }^{B}$ on behalf of the British Society of Gastroenterology Trainees Section
}

\begin{abstract}
Higher specialist training in general internal medicine (GIM) and the medical specialties has been subject to many changes and increasing subspecialisation in recent years. The 'Shape of Training' review proposes 'broad-based specialty training', shortening of training by one year, and subspecialisation to be undertaken after the certificate of specialty training is obtained. All higher level gastroenterology trainees based in the UK were invited to complete an online survey between July and September 2012 to assess their experience of gastroenterology and GIM training. Overall, $72.7 \%$ of trainees expressed satisfaction with their training in gastroenterology but significantly fewer (43.5\%) expressed satisfaction with their training in GIM. Satisfaction with gastroenterology training thus is good, but satisfaction with GIM training is lower and levels of dissatisfaction have increased significantly since 2008 . Up to $50 \%$ of trainees are not achieving the minimum recommended number of colonoscopy procedures for their stage of training. Experience in GIM is seen as service orientated, with a lack of training opportunities. There is a worrying difficulty in gaining the minimum required experience in endoscopy. If the length of specialist training is shortened and generalised, training in key core specialist skills such as endoscopy may be compromised further.
\end{abstract}

KEYWORDS: Endoscopy, gastroenterology, general internal medicine, GIM, training

\section{Introduction}

Higher specialist training in both general internal medicine (GIM) and the medical specialties has been subject to many changes in recent years. Increasing demand on medical registrars' time, both during periods on call and while attempting to attain focused competencies, have been further challenged by a reduction in training time available following the introduction of the European Working Time Directive (EWTD). ${ }^{1,2}$

Authors: A consultant gastroenterologist, Department of Gastroenterology, South Devon Healthcare NHS Foundation Trust, Torbay, Devon, UK; ${ }^{B}$ gastroenterology registrar, Department of Gastroenterology, Southampton General Hospital, Southampton, UK
The reduction in time available for training has impacted on both the confidence and competence of medical trainees at all levels and is therefore a real concern. This has led to increasing stresses for medical registrars, whose service and supervisory roles continue to increase. ${ }^{3}$ Junior medical trainees report a high level of anxiety when contemplating entering higher medical training and an overall view of a poor work-life balance for those already within the medical registrar ranks. ${ }^{4,5}$ In addition, numerous changes have recently been made to training methods and assessment, with the introduction of workplace-based assessments and competency-based curricula. ${ }^{6,7}$ Attaining adequate training both in GIM and in the competencies defined in the curriculum within these boundaries is increasingly challenging. Medical specialties continue to expand, with current trainees able to pursue interests in a wide variety of subspecialist areas that require focused training and attainment of advanced competencies.

The recently published 'Shape of Training' review proposes more broad-based general training leading to a certificate of specialty training (CST). ${ }^{8}$ The review recommends shortening of specialty training by one year and that further subspecialty training is obtained through a system of 'credentialing' undertaken after the CST is obtained. Credentialing would be guided by local demographics and patient needs. Concerns have been raised that these proposed changes may not allow specialty trainees to acquire the core skills needed for their specialty within the proposed training timeframe. ${ }^{9}$ In line with Shape of Training, the Royal College of Physicians' Future Hospital Commission report published in 2013 suggests that 'a greater proportion of doctors will be trained and deployed to deliver expert [general] internal medicine care'. ${ }^{10}$ Currently, these increasing pressures on higher medical trainees' limited time mean that the quality of both GIM and specialty training is under strain.

Every two years, the British Society of Gastroenterology (BSG)'s Trainees Section (formerly Trainees in Gastroenterology) performs a comprehensive survey of all higher level specialty trainees (ST3-7 and specialty registrars (StRs)) in gastroenterology in the UK. The aim of these surveys is to obtain trainees' opinions on a number of aspects of both GIM and specialist training. Previous surveys were carried out in 2008 and 2010. This paper presents the findings of the latest national training survey performed in 2012. 


\section{Methods}

An online survey was designed by the BSG's Trainees Section to assess trainees' current training status, training opportunities and satisfaction with training in GIM and gastroenterology. Views about future careers plans and out-of-programme experience were also assessed.

A pilot survey was trialled with a small sample of trainees to ensure it was user friendly and could be completed in a reasonable time (15-20 minutes). The final survey was open for completion over a three-month period from July to September 2012. Trainees were invited to complete the survey via the BSG's membership database of trainees and regional trainee representatives. Notification and email reminders were sent to all trainees registered in the UK. Data were analysed using Excel (Microsoft, UK).

\section{Results}

\section{Demographics}

Overall, 314 of 790 trainees in the UK responded, giving a response rate of $39.7 \%$, which compares favourably with response rates in previous surveys (35.7\% in 2010 and $52.1 \%$ in $2008)$. Of the responding trainees, $31.1 \%$ were female compared with $34 \%$ in the workforce report of 2011. The vast majority of trainees were following Modernising Medical Careers (MMC) training pathways, with only $12.2 \%$ of respondents in the old specialist registrar $(\mathrm{SpR})$ grade.

\section{GIM training}

Training in GIM remains strongly associated with gastroenterology higher specialist training, with $97.7 \%$ of trainees reporting dual accreditation in gastroenterology and GIM, which is very similar to findings of previous surveys (98.0\% of trainees in 2010 and $97.3 \%$ in 2008). Specialty gastroenterology trainees also recognise the importance of GIM for their future careers, with $80.4 \%$ agreeing ('strongly agree' or 'agree') that GIM experience is important for work as a consultant, $6.8 \%$ of respondents disagreeing ('strongly disagree' or 'disagree'), and $12.7 \%$ being neutral or unsure.

However, there is dissatisfaction regarding the quality of experience obtained through work and training in GIM. Overall, $22.6 \%$ of trainees expressed dissatisfaction with the quality of GIM training received during their higher specialist training to date, only $43.5 \%$ expressed satisfaction, and $31.5 \%$ were neutral (neither satisfied nor dissatisfied). This level of dissatisfaction is much worse than that expressed with regard to specialist gastroenterology training, with only $7.3 \%$ of trainees reporting dissatisfaction with gastroenterology training; this difference is statistically significant when examined using Pearson's $\chi^{2}$ test $(\mathrm{p}<0.01)$. Furthermore, the level of dissatisfaction with GIM training represents an increase on that reported by gastroenterology trainees in previous surveys: $16.7 \%$ in 2010 and $14 \%$ in 2008 . When analysed using Pearson's $\chi^{2}$ test, this increase in dissatisfaction is statistically significant $(\mathrm{p}=0.012)$. By comparison, dissatisfaction with gastroenterology training has remained stable: $7.4 \%$ of respondents expressed dissatisfaction with gastroenterology training in 2010 and $11.0 \%$ in 2008 , with no statistical difference when examined using Pearson's $\chi^{2}$ test $(p=0.18)$.
When asked to specify reasons for dissatisfaction with GIM training, most respondents cited the large service commitment of GIM and the lack of formal training. The service commitment was specifically said to be increasing due to registrar-directed care pathways (eg stroke/acute coronary syndrome) and less-experienced junior colleagues requiring more support in both acute medicine and specialties such as surgery, trauma, orthopaedics and even the emergency department. The lack of formal structure in both the acute medical team and the training provided was also commonly cited. Fig 1 summarises the responses to this question.

Most trainees (55.8\%) estimated that GIM work accounts for $30-50 \%$ of their clinical time. Overall, $56.3 \%$ of respondents $(62.1 \%$ in 2010$)$ felt that the GIM component of their work had a negative impact on them attaining the competencies required in their core specialty training for gastroenterology. Despite this, most trainees (57.5\%) would not discontinue GIM training if given the opportunity to do so, although $27.1 \%$ said that they would and $15.4 \%$ were unsure.

\section{Training in gastroenterology}

Overall, $72.7 \%$ of trainees expressed satisfaction ('satisfied' or 'very satisfied') with their overall training in gastroenterology, $20 \%$ were 'neutral' and $7.3 \%$ expressed dissatisfaction ('dissatisfied' or 'very dissatisfied') (Fig 2).

When analysis was performed by specific areas of the gastroenterology curriculum, training satisfaction was lowest for nutrition, with $26.5 \%$ of all trainees expressing dissatisfaction. In comparison, $18 \%$ of all trainees expressed dissatisfaction with endoscopy training, $14 \%$ with hepatology training and $11 \%$ with inflammatory bowel disease (IBD) training. Overall, $79.2 \%$ of trainees agreed that their current educational supervisor provided adequate support and direction, $15.8 \%$ were neutral and $3.9 \%$ disagreed.

\section{Plans for after certificate of completion of training}

Most trainees expressed an interest in obtaining a general gastroenterology consultant post with an additional interest $(41.6 \%)$ after obtaining their certificate of completion of training (CCT), while $14.3 \%$ plan to work as a 'general gastroenterologist' and $32.9 \%$ expressed ambitions for subspecialty consultant posts, with $14.6 \%$ aiming to specialise in hepatology, $7.7 \%$ in advanced endoscopy, $7.0 \%$ in IBD and $1.4 \%$ in nutrition. Trainees remain uncertain regarding future career prospects upon attaining their CCT, with only $24.6 \%$ confident or very confident that they will obtain their desired job by the end of their training, $29.1 \%$ unconfident/extremely unconfident and $43.9 \%$ neutral. Reflecting these findings, most trainees would consider post-CCT fellowships (81.4\%) or locum appointments $(81.8 \%)$ to gain further experience and enhance their prospects of achieving a substantive appointment.

\section{Endoscopy}

Overall, 95\% of trainees are registered on the online endoscopic procedure documentation system website of the Joint Advisory Group (JAG) Endoscopy Training System (JETS). Of these, $68 \%$ believe JETS has improved endoscopy training and certification, $10 \%$ disagree and $22 \%$ are neutral. 


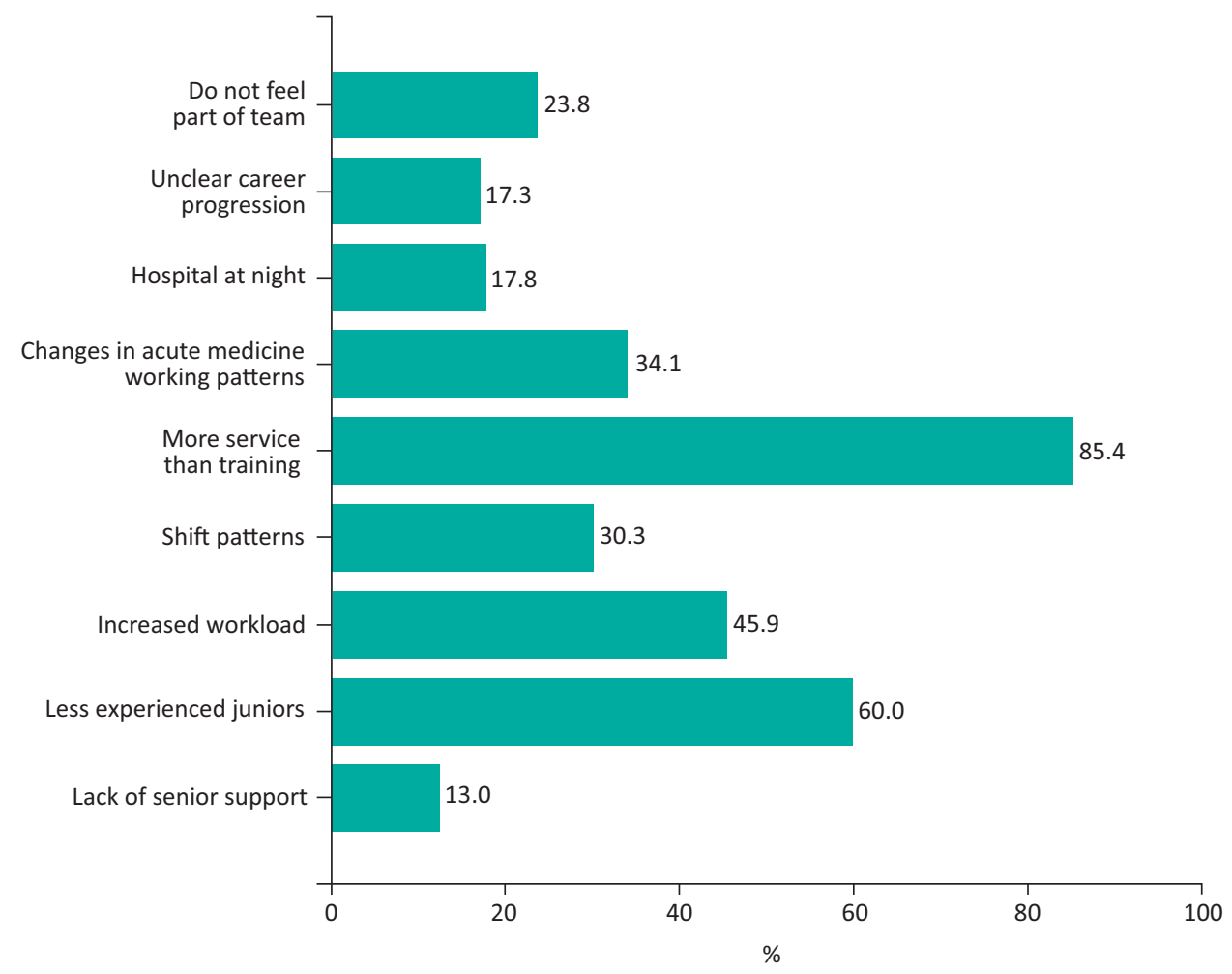

Fig 1. Gastroenterology trainees' responses to specific concerns about experience in general internal medicine. Proportion of responding trainees given (multiple answers allowed).
As Fig 3 demonstrates, the percentage of trainees who have achieved JAG certification for diagnostic gastroscopy rises from $38.2 \%$ among ST3 trainees to $78.7 \%$ among ST4 trainees. However, there is no further notable increase in higher years (74.5\%, 82.9\% and $81.8 \%$ for ST5, ST6 and ST7/8 and StR levels, respectively). Progression to full JAG certification in colonoscopy is slower, with $10.4 \%$ of ST5 trainees being fully JAG certified, rising to $28.6 \%$ of ST6 trainees and $50 \%$ of ST7/8 trainees and StRs.

In general, the median number of diagnostic procedures performed by trainees meets the minimum numbers set out in the gastroenterology annual review of competence progression (ARCP) decision aid. ${ }^{12}$ However, further analysis of the data shows that $16 \%, 32 \%, 26 \%$ and $50 \%$ of ST 4 , ST5,
ST6 and ST7-8/StR trainees, respectively, are not achieving the minimum recommended number of colonoscopy procedures for their stage of training. Trainees close to CCT (ST7-8 and StRs) have performed a median of 800 oesophago-gastroduodenoscopies, 200 flexible sigmoidoscopies and 335 colonoscopies (Fig 4).

Overall, $31 \%$ of trainees view annual leave and rota days off as time to gain further training in endoscopy. The main reported barriers to general endoscopy training were service commitment (64\%), impact of GIM training (62\%) and impact of EWTD (53\%). However, other important factors included lack of training opportunities (45\%), poor-quality training lists $(34 \%)$, poor-quality trainers $(10 \%)$ and course costs $(34 \%)$.

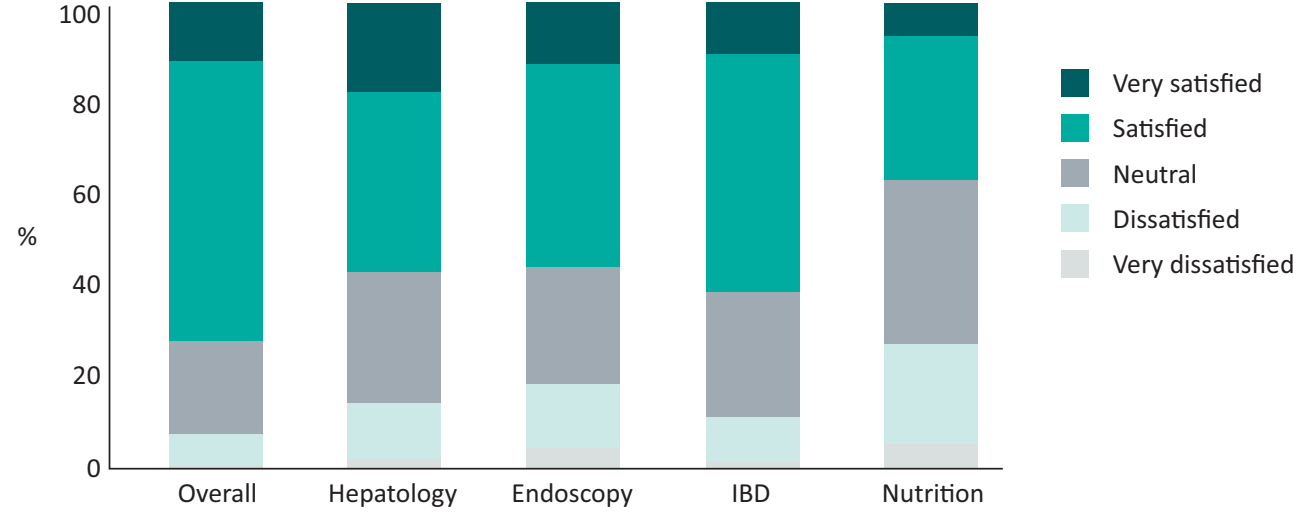

Fig 2. Responses to question 'How would you rate your overall satisfaction with the standard of your training in gastroenterology?' IBD = inflammatory bowel disease. 
Fig 3. Percentage of trainees who obtained JAG certification by training grade. Flexible/provisional colonoscopy $=$ provisional colonoscopy certification plus level 1 polypectomy; full colonoscopy $=$ full colonos copy certification + level 2 polypectomy; oesophagogastro-duodenoscopy = diagnostic upper gastrointestinal endoscopy. JAG = joint advisory group; $\mathrm{SpR}=$ specialty registrar; $\mathrm{ST}=$ specialty training.

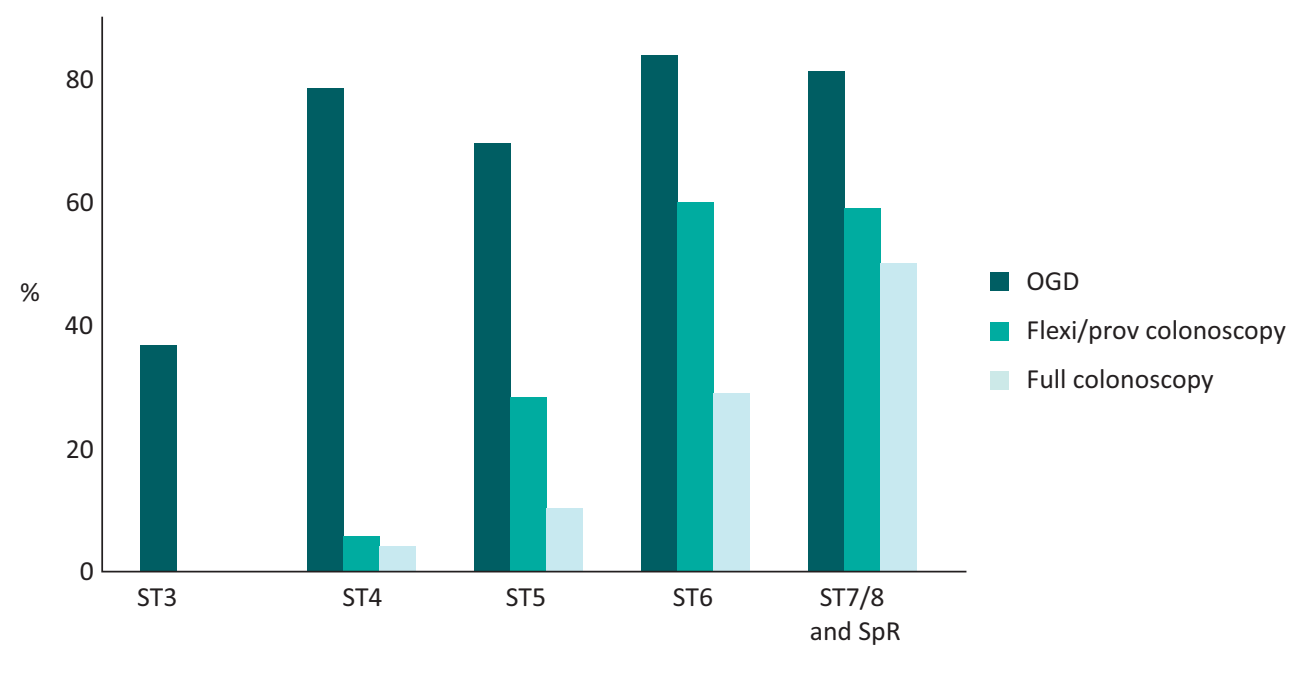

\section{Discussion}

The results of this national survey of gastroenterology trainees provide clear insight into the difficulties higher specialist trainees face attaining high-quality GIM training. Increasing dissatisfaction in GIM work and training is evident from three separate surveys. This dissatisfaction is not reflected in trainees' feelings about specialist training; however, trainees do feel pressure on their specialist training from the increasing GIM workload, and most believe that its impact is negative. Gastroenterology trainees acknowledge the importance of general medicine, continue to actively pursue CCTs in GIM, and see GIM as important to their future work as consultants.

When asked to specify concerns about the quality of GIM training, a number of key themes emerge. First, the workload placed on the on-call medical registrar is increasing from a number of avenues. ${ }^{4,5}$ The reduction in senior cover out of hours for other specialties, such as surgery and, in some places, the emergency department, means the medical

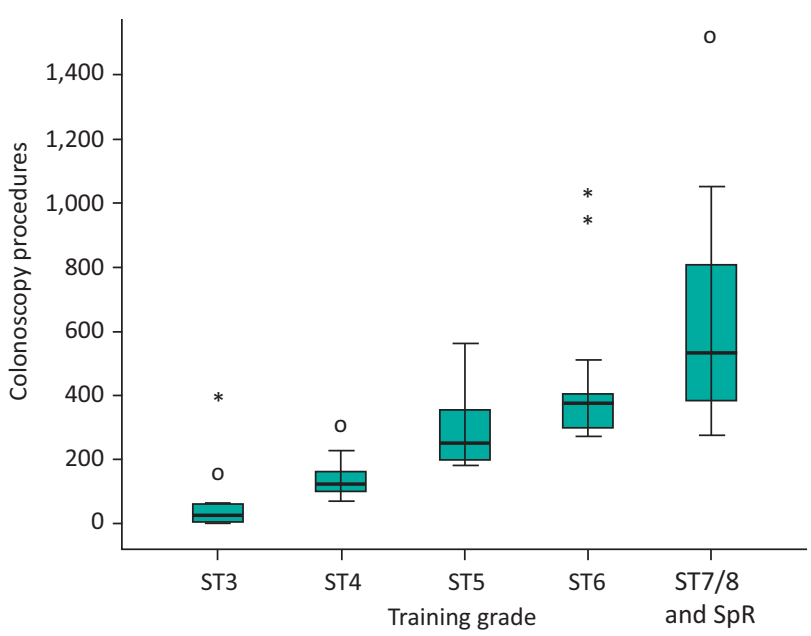

Fig 4. Box plot of colonoscopy procedures performed by training grade. Dark horizontal line indicates median. Shaded box indicates interquartile range. Whiskers show top and bottom $25 \%$. Circles/stars indicate outliers. SpR = specialty registrar; $\mathrm{ST}=$ specialty training. registrar becomes responsible for this support. ${ }^{5,13}$ This is in conjunction with a perceived reduction in the number and ability of junior medical trainees, ${ }^{14}$ which leads to increases in both the educational and practical support required. However, increasing time pressure means that many medical registrars are left frustrated at the quality of support they can provide. ${ }^{5}$

Second, experience in GIM is overwhelmingly seen as service orientated, with a lack of training opportunities. Registrars feel a lack of a clearly structured team, and opportunities to discuss cases in a protected fashion are very limited.

Finally, underpinning these concerns are the changes in work patterns, which have, in part, been introduced following the introduction of the EWTD. The impact of shift patterns on both specialist training and work-life balance are felt strongly by registrars.

The relative dissatisfaction in GIM training compared with specialist training was demonstrated previously by the National Registrars' Survey, performed by the Royal College of Physicians (RCP)'s Medical Workforce Unit. ${ }^{15}$ In 2010, a very similar overall level of satisfaction in GIM was recorded, with $48.6 \%$ of the 2,176 responding registrars satisfied with GIM training; this compares with $43.5 \%$ in our survey, which used the same five-point satisfaction scale. In the RCP's survey, $88.0 \%$ of respondents expressed satisfaction with specialty training compared with $72.7 \%$ of respondents in our survey. Both of these sets of results highlight the gap between specialty medical training and GIM training.

Gastroenterology and other medical specialties are increasingly diverse fields, and many new consultants have at least one specialty area of expertise. ${ }^{3}$ This is clearly recognised by gastroenterology trainees, as only $14.3 \%$ plan to work as a 'general gastroenterologist' after they obtain their CCT, with the remainder planning to work as a subspecialist or general gastroenterologist with a specialty interest.

From 2011, trainees who want to specialise in nutrition, IBD or advanced endoscopy undertake a focused year of training in their chosen area, known as an advanced training module (ATM), ${ }^{7}$ usually at ST6 level. Hepatology subspecialty training is now incorporated within ATMs. The recent Shape of Training review could mean that this period of specialty training moves from being incorporated within registrar training to after the proposed CST. ${ }^{8}$ The above results suggest that the next 
generation of gastroenterologists aspire to work fully or partly in an area of specialty interest at a time when it is suggested that we need more generalists. ${ }^{10} \mathrm{With}$ the increasing diversification and subspecialisation of other medical specialties, similar issues are likely to arise for other physician trainees. Whether opportunities to develop subspecialty skills after the CST will be available may depend largely on the views of local employers and commissioners. ${ }^{14}$ The impact of these proposed changes on morale, recruitment and retention of higher level physician trainees needs to be considered by policy makers.

Progression of gastroenterology trainees through basic endoscopy training and certification is an area of concern, with a sixth of ST6 trainees not certified for diagnostic gastroscopy and just over a quarter at this level having obtained full colonoscopy certification. Service commitment, GIM training commitments and the EWTD are the main identified barriers to training in endoscopy, and the combined effect of these three factors may account for what seems to be slower progress towards achieving competence in endoscopy than in the past. Evidence indicates that trainees are trying to alleviate this by performing endoscopy outside of their contracted hours. The proposed shortening of higher specialty training time by one year and increased 'generalisation' of training may impact further on the ability of gastroenterology trainees to acquire the core endoscopic skills required by the time they reach CST. ${ }^{8}$ Similar issues are likely to arise for higher level trainees in other medical specialties and could lead to a cohort of holders of CSTs who are not equipped to function as fully independent consultants.

In summary, trainee satisfaction in general medicine seems to be diminishing due to increasing service commitments and lack of well-defined training. Increasing pressures on higher specialty trainees seems to be impacting negatively on specialty training, particularly in terms of achieving competence in core specialist skills such as endoscopy. This survey demonstrates these issues among gastroenterology trainees and highlights the need for further debate about how to educate and recruit the next generation of physicians.

\section{Acknowledgements}

JN designed and rolled out the survey, analysed the results, and drafted and revised the article. PB analysed the results and revised the article. The BSG's Trainees Committee commissioned the survey and revised the article.

\section{Funding}

Costs associated with the survey were met by the BSG's Trainees Committee. No external funding was received.

\section{References}

1 Bates T, Cecil E, Green I. The effect of the EWTD on training in general surgery: an analysis of electronic logbook records. Bull $R$ Coll Surg Engl 2007;89:106-9.

2 Sim DJ, Wrigley SR, Harris S. Effects of the European Working Time Directive on anaesthetic training in the United Kingdom. Anaesthesia 2004;59:781-4.

3 Morris AI. Changing practice in gastroenterology in a changing environment. Clin Med 2007;7:551-3.

4 Chaudhuri E, Mason NC, Newbery N, Goddard AF. Career choices of junior doctors: is the physician an endangered species? Clin Med 2013;13:330-5.

5 Chaudhuri E, Mason NC, Logan S, Newbery N, Goddard AF. The medical registrar: empowering the unsung heroes of patient care. London: Royal College of Physicians, 2013.

6 Joint Royal Colleges of Physicians Training Board. Workplace based assessments. Available online at www.jrcptb.org.uk/assessment/Pages/ Workplace-Based-Assessment.aspx [Accessed 26 November 2014].

7 Joint Royal Colleges of Physicians Training Board. Specialty training curriculum for gastroenterology: August 2010. London: JRCPTB, 2010. Available online at http://www.gmc-uk.org/Gastroenterology_ curriculum_2010.pdf_32486121.pdf_43285174.pdf [Accessed 26 November 2014].

8 Shape of Training. Securing the future of excellent patient care: final report of the independent review led by Professor David Greenaway. Available online at www.shapeoftraining.co.uk/static/documents/ content/Shape_of_training_FINAL_Report.pdf_53977887.pdf [Accessed 26 November 2014].

9 Fuller G, Simpson I. 'Modernising Medical Careers' to 'Shape of Training' - how soon we forget. BMJ 2014;348:g2865.

10 Royal College of Physicians. Future hospital commission. Available online at www.rcplondon.ac.uk/projects/future-hospital-commission [Accessed 28 November 2014].

11 Gordon H, Romaya C. BSG workforce report 2011. London: British Society of Gastroenterology, 2011. Available online at www.bsg.org. uk/training/general/training-committee.html [Accessed 19-12-2014]

12 Joint Royal Colleges of Physicians Training Board. Gastroenterology ARCP decision aid. Available online at www.jrcptb.org.uk/sites/ default/files/2010\%20Gastroenterology\%20ARCP\%20Decision $\% 20$ Aid\%20\%28revised\%202014\%29.pdf [Accessed 28 November 2014].

13 Mason NC, Chaudhuri E, Newbery N, Goddard AF. Training in general medicine - are juniors getting enough experience? Clin Med 2013;13:434-9.

14 Goddard AF, Evans T, Phillips C. Medical registrars in 2010: experience and expectations of the future consultant physicians of the UK. Clin Med 2011;11:532-5.

15 Hodgson $\mathrm{H}$. The shape of training - a flexible friend? Clin Med 2014;14:3.

Address for correspondence: Dr PJ Basford, British Society of Gastroenterology, 3 St Andrews Place, Regent's Park, London NW1 4LB, UK.

Email: petebasford@hotmail.com 